\title{
Perceptual and motor congruency effects in time-space association
}

\author{
Marco Fabbri • Nicola Cellini • Monica Martoni • \\ Lorenzo Tonetti • Vincenzo Natale
}

Published online: 1 August 2013

(C) Psychonomic Society, Inc. 2013

\begin{abstract}
It is well established that temporal events are represented on a spatially oriented mental time line from left to right. Depending on the task characteristics, the spatial representation of time may be linked to different types of dimensions, including manual response codes and physical space codes. The aim of the present study was to analyze whether manual response and physical space codes are independent of each other or whether they interact when both types of information are involved in the task. The participants performed a temporal estimation task with two lateralized response buttons in four experiments. In the first experiment, the target stimuli were presented on the left side, at the center, or on the right side of the space, whereas the reference stimuli were always presented centrally. The reverse situation was presented in the second experiment. In the third experiment, both stimuli were presented in opposite spatial positions (e.g., left-right), whereas in the last experiment, both stimuli were presented in the same spatial position (e.g., left-left). In all experiments, perceptual and motor congruency effects were found, but no modulation of the congruency effects was found when both the perceptual and motor components were congruent. The results indicated that physical, spatial, and manual response codes are independent from each other for
\end{abstract}

M. Fabbri $(\triangle)$

Department of Psychology, Second University of Naples, Viale

Ellittico, 31, 81100 Caserta, Italy

e-mail: marco.fabbri@unina2.it

N. Cellini

Department of General Psychology, University of Padua, Padua, Italy

\section{Martoni}

Department of Experimental, Diagnostic, and Specialty Medicine, University of Bologna, Bologna, Italy

L. Tonetti $\cdot$ V. Natale

Department of Psychology, University of Bologna, Bologna, Italy time-space associations, even when both codes are involved in the task. These results are discussed in terms of the "intermediate-coding" account.

Keywords STEARC effect - Spatial processing - Spatialtemporal association $\cdot$ Temporal estimation task $\cdot$ Mental time line

Cognitive psychology has addressed the issue of how abstract concepts, such as time, are represented in memory. It has been suggested that abstract concepts are coded by means of more concrete domains, such as space. According to the conceptual metaphor theory (Lakoff \& Johnson, 1980, 1999), in many languages, the abstract concept of time is naturally associated with a horizontal back-to-front metaphor, which maps the past to locations in the back, the future to locations in the front, and time passing to forward movement (for a review, see Radden, 2004). For instance, Torralbo, Santiago, and Lupiáñez (2006) asked participants to judge whether a word (e.g., yesterday or tomorrow) appearing at the front or the back of a side-looking head silhouette referred to the past or to the future. The participants responded more quickly when past words were presented at the back of the head and future words were presented at the front.

When a left-right manual response was used, Torralbo et al. (2006) also showed that responses were faster when the past words were judged with the left key and future words were judged with the right key than when an opposite wordkey assignment was provided. This finding supports the idea that time can be represented using a left-to-right mental representation. Further evidence for a spatial representation of time can be found in Weger and Pratt's (2008) study, in which participants responded more quickly using a left-toright representation of time when they had to discriminate whether an actor/actress (e.g., Marilyn Monroe vs. Kate 
Winslet) became popular before or after participants were born. Recently, Santiago, Román, Ouellet, Rodríguez, and Pérez-Azor (2010) extended the spatial mapping of time to experienced event sequences presented by video clips or photograph sequences. Order judgments of two events were shorter for the left hand to respond "before" and the right hand to respond "after" than with the opposite pattern. These data are consistent with the observation that American children ordered temporal concepts from left to right, whereas Arabic children showed a right-to-left order when associating events in time (e.g., breakfast, lunch, and dinner times) with locations in space (e.g., three stickers on a piece of paper; Tversky, Kugelmass, \& Winter, 1991). Altogether, the pattern of these results suggests that elapsing time is internally mapped onto spatial representations (for a review, see Bonato, Zorzi, \& Umiltà, 2012). This time-space mapping seems to support the idea of a mental time line, in which temporal events are spatially represented on a temporal line oriented from left to right (Arzy, Adi-Japha, \& Blanke, 2009).

Left-to-right temporal mapping could be accounted for in two different ways. On the one hand, temporal stimuli (e.g., yesterday or tomorrow) seem to direct spatial attention according to the spatial position of temporal information along the time line. According to this account, Torralbo et al. (2006) presented words referring to the past or the future at different spatial locations (e.g., left-right). Responses were faster when the task-irrelevant word location was congruent with the left-past and right-future metaphoric mapping. On the other hand, temporal stimuli prime motor responses to left and right sides of space according to the spatial congruency between temporal information on the time line and the positions of response buttons (i.e., congruent conditions are defined when short durations are associated with a left button, whereas long durations are associated with a right button). In a recent study (Rolke et al., 2013, Exp. 1), the task was to indicate the color (pink or yellow) of a central target square using bimanual response. Before the presentation of the square, a past (i.e., yesterday) or future (i.e., tomorrow) word was visually presented at the center of the screen. The authors obtained a significant time-space congruency effect, with faster left-hand responses when the square followed a past word and faster right-hand responses when the square followed a future word.

Santiago, Lupiáñez, Pérez, and Funes (2007) presented temporal stimuli (e.g., before or after) and the response keys on the left or right side of space. The authors found that judgments were facilitated when the word position or response mapping was congruent with the left-past and right-future metaphoric mapping. Ouellet, Santiago, Funes, and Lupiáñez (2010) asked participants to perform a spatial Stroop task while keeping in mind the temporal value of a cue (past or future). The results showed that the activation of temporal references (past or future) both influenced the orientation of spatial attention and primed motor responses to the left or right of space. Nevertheless, the authors stated that the perceptual and motor levels were independent of each other, in line with the results of Santiago et al. (2007).

Kong and You (2012) proposed the "intermediate-coding" account, which states that a temporal stimulus, such as a time-related word, is first categorized as "past" or "future," and then this "temporal" concept activates an abstract spatial code referring to "left" or "right," respectively. This abstract spatial code could prime a left-right manual response code (i.e., the motor level) or influence the orientation of spatial attention (i.e., the perceptual level). The activation of response or physical space codes would depend on the task characteristics, such as a bimanual response task or spatially located stimuli. When manual responses and physical space codes are involved in the task at the same time, the intermediate-coding account states that the left-to-right representation of time would be associated with each spatial dimension of the task independently.

Given that the use of temporal words might facilitate spatial biases due to the directional action of reading (Tversky et al., 1991), putting in question the automatic activation of spatial-temporal associations, in some studies researchers have investigated space-time interactions using a more basic dimension of time - that is, temporal duration, which is also the focus of the present study. For example, Vallesi, Binns, and Shallice (2008) asked participants to judge the duration (e.g., 1 or 3 s) of a central cross by pressing two lateralized response buttons. The participants were faster if they responded to short durations using the leftmost response and long durations using the rightmost response, rather than the reverse mapping. Similarly, the results from time estimation task studies with manual responses in which participants were asked to press one of two response keys, depending on whether the timing of a given probe was earlier or later than would be expected on the basis of the preceding clicks, have shown an association between left responses and early onset timing and between right responses and late onset timing (Ishihara, Keller, Rossetti, \& Prinz, 2008). Both studies have yielded a timespace interaction reflecting a spatial-temporal association of response codes (STEARC) effect, in which short temporal durations are represented on the left and long temporal durations on the right side of space, in a left-to-right mapping (see also Fabbri, Cancellieri, \& Natale, 2012).

The STEARC effect reflects the role of motor components in associating space with time. In a temporal estimation task with two lateralized response keys, Fabbri et al. (2012) found a significant STEARC effect when they presented both reference and target stimuli in a central position (see also Vallesi et al., 2008). The associations between left space and short durations and between right space and long durations could 
only be explained by considering the role of spatial (leftright) response keys in representing time, given that the spatial information of stimuli was fixed in central space. This assumption has recently been corroborated with the finding of the STEARC effect in a temporal reproduction task (Fabbri, Cellini, Martoni, Tonetti, \& Natale, 2013). Indeed, the STEARC effect, but not the congruency effect between durations and stimulus locations, was found in all experiments, reflecting that short durations were reproduced better with the left key, whereas long durations were reproduced better with the right key, even if the temporal stimuli to be reproduced appeared at different spatial positions on the screen.

Recently, Di Bono et al. (2012) asked participants to orally respond in a duration judgment (short-long) task to auditory stimuli (e.g., tones), while irrelevant visuospatial primes were presented either on the left or the right side of the display. In this experimental procedure, the authors investigated whether visuospatial processing influenced the spatial-temporal association. When the auditory target was paired with left-side visual primes, the responses to short durations were faster than those when the target was paired with a right-side prime. Contrarily, the responses to long durations were faster when the target was paired with rightside rather than left-side primes. This finding supports the idea of a left-to-right time representation. Importantly, the responses were not spatially characterized (instead, they were verbally provided), thus ruling out the possibility that the effect was due to a facilitation of response codes. Without the influence of response codes, the left-to-right time representation seems to be influenced by the spatial information of visual primes (but see Exp. 2 of Vicario, Rappo, Pepi, \& Oliveri, 2009, for different results).

The intermediate-coding account (Kong \& You, 2012) seems to explain the spatial-temporal association, even when temporal durations are used. The influence of motor or perceptual components in the left-to-right mapping of time depends on the specific experimental setting (i.e., the involvement, or lack thereof, of manual responses in the task) and task demands. However, it remains to be clarified what happens when both temporal stimuli and manual responses are spatially coded. Vicario et al. (2008) found that participants' judgments of temporal duration were influenced by the horizontal spatial location of stimuli (digit or dots). Specifically, left space biased estimations toward short durations, whereas right space biased estimations toward long durations. Although responses were also spatially coded with a left-right buttonpress, Vicario et al. (2008) did not analyze any possible STEARC effect. In a similar experimental procedure, Fabbri et al. (2013) did not find any estimation biases of temporal reproduction based on the spatial information provided by the stimulus positions on the screen. However, the authors found that the temporal reproduction was influenced by the spatial information provided by the response keys, with better performance when short durations were reproduced using the left key and long durations were reproduced using the right key, than with the opposite pairings. However, in the temporal reproduction task, in which temporal judgments are based more on motor than on perceptual representations (Bueti \& Walsh, 2010; Fabbri et al., 2012), the motor components might have masked (or reduced) the contribution of the perceptual components when manual responses and physical spatial codes were involved in the task at the same time. This last assumption is based on the main difference between temporal estimation and reproduction tasks. Both tasks require a motor response, with the difference that in the temporal estimation task the motor response is a time judgment (i.e., shorter-longer), whereas in the temporal reproduction task, the motor response reproduces an estimated duration - and thus, action duration is coded as part of a motor program and can be processed prior to motor execution (e.g., Vidal, Bonnet, \& Macar, 1991). This feature of temporal reproduction fits with Lewis and Miall's (2006) theory stating that tasks that require reproduction of a temporal duration via an action may be especially reliant on the motor system.

The aim of the present study was to investigate the influences of perceptual and motor components in space-time interactions using a visual temporal estimation task with two lateralized response buttons. We created four experiments, modifying and combining the spatial position of the reference and target stimuli. In Experiments 1 and 2, only one stimulus (reference or target) spatially varied along the screen, while the other remained fixed in the central position. In Experiments 3 and 4, both stimuli varied spatially.

\section{Experiment 1}

In Experiment 1, participants performed a temporal estimation task with subsecond intervals. In particular, the reference stimulus (a yellow rectangle) was always presented at the center of the screen, whereas the target stimulus (a light blue rectangle) could appear in one of three spatial positions: at left, center, or right locations on the screen. The participants performed the task twice, with two lateralized response buttons, allowing for measurement of the STEARC effect (Ishihara et al., 2008). Thus, two spatial aspects were provided: the spatial information of the response buttons, and that of the target position.

The interaction between the spatial position of response keys and the duration judgments (short-long) should indicate a STEARC effect (Ishihara et al., 2008), suggesting the contribution of response components in associating space with time. The interaction between the spatial position of stimuli and the duration judgments should indicate a Duration $\times$ Stimulus Location congruency effect (Santiago et al., 2007; Torralbo et al., 2006), suggesting the contribution of a 
perceptual component in spatial-temporal associations. Finally, the triple interaction between the spatial positions of both the response keys and the target stimuli and the duration judgments should indicate that both perceptual and motor levels contribute to spatial-temporal associations. In other words, the triple interaction should indicate a modulation of the congruency effect when the motor and perceptual components are congruent (i.e., left key, left space, and short durations and right key, right space, and long durations).

\section{Method}

Participants A group of 20 university students (17 females, three males) volunteered to participate in the experiment. Their mean age was 23.60 years $(S D=1.23)$. The participants filled in the Edinburgh Handedness Inventory (EHI; Oldfield, 1971). According to the EHI scores, 17 were righthanded and three were left-handed. All of the participants had normal or corrected-to-normal vision. The study was conducted following university ethical guidelines, and all participants provided written informed consent.

Materials All stimuli were presented on the dark background of a computer screen. The display had a resolution of $1,072 \times 960$ pixels and was refreshed at a rate of $72 \mathrm{~Hz}$. All participants sat facing the computer screen at a viewing distance of $60 \mathrm{~cm}$. The stimuli consisted of two rectangles in a temporal estimation task. The reference stimulus was a yellow rectangle (192 pixels in length $\times 128$ pixels in height), whereas the target stimulus was a light-blue rectangle (192 pixels in length $\times 128$ pixels in height). The reference-target pairs determined three spatial positions: (1) center-left $(\mathrm{C}-\mathrm{L}),(2)$ center-center $(\mathrm{C}-\mathrm{C})$, or (3) center-right (C-R) position. The $x$ - and $y$-coordinates for the left, center, and right positions were, respectively, (a) $256 \times$ 384 pixels, (b) $512 \times 384$ pixels (these coordinates were equal for the yellow and light-blue rectangles), and (c) $768 \times 384$ pixels. The coordinates indicate the centers of the rectangles.

Procedure All participants were individually tested in a quiet room. Stimulus presentation and data collection were controlled using E-Prime 1.1 (Schneider, Eschman, \& Zuccolotto, 2002). The participants were required to judge whether the duration of a target stimulus (light-blue rectangle) appearing on the screen was shorter or longer than that of a reference stimulus (yellow rectangle). The duration of the reference cue was fixed across the trials, with the yellow rectangle remaining on screen for $400 \mathrm{~ms}$. The duration of the target could range from 200 to $600 \mathrm{~ms}$ in steps of $100 \mathrm{~ms}$, with the exception of $400 \mathrm{~ms}$. The durations of 200 and $300 \mathrm{~ms}$ were considered short durations, whereas the durations of 500 and $600 \mathrm{~ms}$ were considered long durations. The response keys were the " 4 " and " 6 " keys of the numerical keypad on a normal keyboard, which were covered by two green discs in order to avoid any numerical influence. The participants had to press the "4" key with their left hand and the "6" key" with their right hand.

Similar to the procedure carried out by Cappelletti, Freeman, and Cipolotti (2009), a white cross $(+)$ sign in Courier New 120-point type appeared at the center of the screen as a fixation point for $400 \mathrm{~ms}$. After the fixation point, a black screen appeared for $200 \mathrm{~ms}$. After that, a yellow rectangle (the reference stimulus) appeared centrally on the screen with a black background. The reference cue lasted $400 \mathrm{~ms}$ in all trials. Then, a black screen appeared as an interstimulus interval (ISI). In order to avoid any response strategy, this black screen lasted for a randomly selected interval between 700 and $800 \mathrm{~ms}$. After this ISI, a light-blue rectangle (the target stimulus) appeared on a black background at the left, central, or right position. The participants were instructed to refrain from making a judgment at this moment until after the presentation of the target stimulus, when a white question mark ("'?") in Courier New 120-point type appeared at the center of the screen on a black background. It was presented with a "beep" sound in order to prompt participants to make a judgment by pressing one of the corresponding keys. The question mark remained on the screen for $1,500 \mathrm{~ms}$ or until the participant's response was made. We stressed to the participants that accuracy, as well as speed, were fundamental. Finally, the new fixation point appeared after a black screen of $400 \mathrm{~ms}$. The task was performed twice, using two different blocks, in which the instruction-key assignments were counterbalanced. In one block, the left key was pressed in order to judge that the light-blue rectangle was shorter in duration, and the right key was pressed to judge that the lightblue rectangle was longer. In the other block, the left key was pressed in order to judge that the target stimulus was longer in duration, and the right key was pressed to judge that the target stimulus was shorter. In each block, 120 trials were presented in a pseudorandom order. Thus, altogether, the participants judged 240 trials. Before the test, a training session was run, with 12 trials presenting all three spatial positions of target stimuli lasting from 200 to $600 \mathrm{~ms}$. The training phase could be performed for a second time if requested by the participants. After each block, individuals had the opportunity to take a 1-min break. The experiment lasted approximately $30 \mathrm{~min}$.

Data analysis The mean reaction times (RTs) of the correct responses were calculated. Moreover, RTs more than $3 S D \mathrm{~s}$ above or below the mean were excluded from the analysis because they were considered outliers (about $2 \%$ of the original sample). To clearly investigate whether the perceptual and/or motor components were independent from each other, or whether they interacted in spatial-temporal associations, a three-way repeated measures analysis of variance 
(ANOVA) was carried out on the RTs, with Key (two levels: left vs. right), Duration (two levels: short vs. long), and Condition (two levels: $\mathrm{C}-\mathrm{L}$ vs. $\mathrm{C}-\mathrm{R}$ ) as within-subjects factors. We decided to exclude the $\mathrm{C}-\mathrm{C}$ condition from analysis because this condition included spatial information only from the response keys, without any spatial perceptual information from the stimuli. The $\mathrm{C}-\mathrm{C}$ condition thus could be used only for testing the presence of the STEARC effect (motor component), but not for testing the presence of the spatial-temporal congruency effect (perceptual component). Therefore, analysis of the $\mathrm{C}-\mathrm{C}$ condition could affect the results and their interpretation. The same ANOVA was carried out on accuracy, as numbers of errors (NEs). When reliable significance was found, the Scheffé post-hoc test was run. Values with $p<.05$ were considered as being significant.

\section{Results and discussion}

Table 1 reports the mean RTs for the spatial response keys and temporal durations at all spatial positions of the reference-target pairs.

The ANOVA ${ }^{1}$ on RTs revealed no main effects $\left(F_{\mathrm{S}}<\right.$ $1.00, p \mathrm{~s}>.55)$. The Key $\times$ Duration interaction was significant, indicating a STEARC effect $\left[F(1,19)=31.79, p<.0001, \eta_{\mathrm{p}}{ }^{2}=\right.$ .63]. The post-hoc test, indeed, showed that for short durations, the left key (548 ms, $S D=66 \mathrm{~ms}$ ) elicited faster RTs than did

\footnotetext{
${ }^{1}$ In the overall ANOVA on RTs with Key (two levels: left vs. right), Duration (two levels: short vs. long), and Condition (three levels: C-L vs. $\mathrm{C}-\mathrm{C}$ vs. $\mathrm{C}-\mathrm{R}$ ) as within-subjects factors, we found a significant condition effect $\left[F(1.582,30.066)=8.68, p<.005, \eta_{\mathrm{p}}{ }^{2}=.31\right.$, with Greenhouse-Geisser correction]. This main effect revealed that the $\mathrm{C}-$ $\mathrm{C}$ condition $(587 \mathrm{~ms}, S D=87 \mathrm{~ms})$ produced higher RTs than did the other two conditions ( $p<.05$ for both comparisons). The Condition factor significantly interacted with the Duration factor $[F(1.901$, $36.122)=5.73, p<.05, \eta_{\mathrm{p}}{ }^{2}=.23$, using Greenhouse-Geisser correction]. A post-hoc test revealed no significant difference between short $(589 \mathrm{~ms}, S D=94 \mathrm{~ms})$ and long $(586 \mathrm{~ms}, S D=80 \mathrm{~ms})$ durations for the $\mathrm{C}-\mathrm{C}$ condition. Also, a significant triple interaction was found $\left[F(1.456,27.655)=4.34, p<.05, \eta_{\mathrm{p}}{ }^{2}=.19\right.$, with Greenhouse-Geisser correction]. Probably, this interaction was reliable due to slower RTs in the $\mathrm{C}-\mathrm{C}$ condition (Table 1). Finally, the Key $\times$ Duration interaction remained reliable.

The same ANOVA on NEs showed a significant condition effect $\left[F(1.507,28.624)=6.34, p<.05, \eta_{\mathrm{p}}{ }^{2}=.25\right.$, with Greenhouse-Geisser correction]. The $\mathrm{C}-\mathrm{C}$ condition $(1.74, S D=1.20)$ elicited higher NEs than did the other two conditions ( $p<.05$ for both comparisons). The Condition factor interacted with the Key factor $[F(1.935,36.763)=$ $3.94, p<.05, \eta_{\mathrm{p}}{ }^{2}=.17$, with Greenhouse-Geisser correction] and the Duration factor $\left[F(1.634,31.041)=5.71, p<.05, \eta_{\mathrm{p}}{ }^{2}=.23\right.$, with Greenhouse-Geisser correction] (Table 2). In the $\mathrm{C}-\mathrm{C}$ condition, no significant difference emerged between right $(1.70, S D=1.06)$ and left $(1.79, S D=1.34)$ keys. When short judgments $(0.69, S D=0.70)$ were required, the participants made smaller NEs than when long judgments were required $(2.80, S D=1.70)$ in the $\mathrm{C}-\mathrm{C}$ condition. No significant triple interaction was found $(F \approx 1.00, p>.30)$, but a significant Key $\times$ Duration interaction was shown.
}

the right key ( $586 \mathrm{~ms}, S D=87 \mathrm{~ms}$ ), with $p<.05$. Moreover, the right key determined lower RTs in judging long durations $(548 \mathrm{~ms}, S D=69 \mathrm{~ms})$ than did the left key $(580 \mathrm{~ms}, S D=$ $79 \mathrm{~ms}$ ), with $p<.05$. The interaction between duration and condition was also significant $\left[F(1,19)=12.88, p<.005, \eta_{\mathrm{p}}{ }^{2}=\right.$ $.40]$, indicating a Durations $\times$ Stimulus Locations congruency effect. The Scheffé test showed that for long durations, the C-R condition (554 ms, $S D=60 \mathrm{~ms}$ ) induced faster responses than did the C-L condition ( $573 \mathrm{~ms}, S D=88 \mathrm{~ms}$ ), with $p<.05$. Even though the result was not significant, the participants responded faster to short durations when the target appeared on the left side (561 ms, $S D=90 \mathrm{~ms}$ ), as compared to right presentation of the target (573 ms, $S D=63 \mathrm{~ms})$. The Key $\times$ Condition interaction did not reach statistical significance $(F<$ $1.00, p>.50)$. Crucially, a triple interaction was not found $[F(1$, 19) $=0.07, p=.79]$, and no modulation of the congruency effect was observed.

In Table 2, the mean NEs are shown for the spatial response keys and temporal durations at all spatial positions of the reference-target pairs.

The aforementioned ANOVA on NEs showed a main duration effect $\left[F(1,19)=22.04, p<.0001, \eta_{\mathrm{p}}{ }^{2}=.54\right]$, reflecting higher accuracy for judging short durations $(0.72, S D=0.80)$ rather than long durations $(2.09, S D=1.38)$. No other main effects were significant $\left(F_{\mathrm{s}}<3.00, p \mathrm{~s}>.10\right)$. The Key factor significantly interacted with both duration $[F(1,19)=7.23$, $\left.p<.05, \eta_{\mathrm{p}}{ }^{2}=.28\right]$ and condition $[F(1,19)=7.69, p<.05$, $\left.\eta_{\mathrm{p}}{ }^{2}=.29\right]$. The former interaction indicated that participants were better at judging short durations $(0.51, S D=0.81)$ than long durations $(2.30, S D=1.48)$ with the left key $(p<.05)$. No other significant comparisons were found. The latter interaction reflected a Simon-like effect, with lower NEs for the C-L condition with the left key $(1.30, S D=1.06)$ than with the right key $(1.64, S D=1.13)$, whereas in the $\mathrm{C}-\mathrm{R}$ condition, accuracy was higher with the right key $(1.18, S D=0.93)$ than with the left key $(1.51, S D=1.23)$. However, no significant comparisons were shown. Finally, the Duration $\times$ Condition interaction, as well as the triple interaction $(F \mathbf{s} \approx 0)$, did not reach statistical significance.

In Experiment 1, we found that the STEARC (Fabbri et al., 2012; Ishihara et al., 2008; Vallesi et al., 2008) and Duration $\times$ Stimulus Location congruency effects (Santiago et al., 2007; Torralbo et al., 2006) were reliable when the RTs were analyzed. These results suggest that motor and perceptual components contribute to spatial-temporal association in independent ways. This assumption was also based on the lack of significant congruency effect between the spatial information provided by both response keys and the $\mathrm{C}$ $\mathrm{L} / \mathrm{C}-\mathrm{R}$ conditions and temporal information provided by the target. The data are in line with the findings of Ouellet et al. (2010), suggesting that temporal judging performance was affected by the spatial positions of the response keys and of target on the screen. In addition, our data seem to be in line 
Table 1 Mean reaction times (in milliseconds, with standard deviations) for left and right keys when judging short and long durations, for each spatial condition in all experiments

\begin{tabular}{|c|c|c|c|c|c|c|}
\hline \multirow[b]{2}{*}{ Experiment 1} & \multicolumn{3}{|c|}{ Short Durations } & \multicolumn{3}{|c|}{ Long Durations } \\
\hline & $\mathrm{C}-\mathrm{L}$ & $\mathrm{C}-\mathrm{C}$ & $\mathrm{C}-\mathrm{R}$ & $\mathrm{C}-\mathrm{L}$ & $\mathrm{C}-\mathrm{C}$ & $\mathrm{C}-\mathrm{R}$ \\
\hline Left key & $540(64)$ & $582(86)$ & $556(67)$ & $589(97)$ & $586(74)$ & $571(60)$ \\
\hline Right key & $582(116)$ & $595(101)$ & $589(58)$ & 558 (79) & $586(85)$ & $538(59)$ \\
\hline Experiment 2 & $\mathrm{~L}-\mathrm{C}$ & $\mathrm{C}-\mathrm{C}$ & $\mathrm{R}-\mathrm{C}$ & $\mathrm{L}-\mathrm{C}$ & $\mathrm{C}-\mathrm{C}$ & $\mathrm{R}-\mathrm{C}$ \\
\hline Left key & $563(47)$ & $608(55)$ & $592(39)$ & 609 (77) & $614(43)$ & $606(47)$ \\
\hline Right key & $591(67)$ & $608(37)$ & $591(53)$ & $584(54)$ & $614(46)$ & $557(60)$ \\
\hline Experiment 3 & $\mathrm{R}-\mathrm{L}$ & $\mathrm{C}-\mathrm{C}$ & $\mathrm{L}-\mathrm{R}$ & $\mathrm{R}-\mathrm{L}$ & $\mathrm{C}-\mathrm{C}$ & $\mathrm{L}-\mathrm{R}$ \\
\hline Left key & $580(166)$ & 647 (174) & $613(163)$ & $663(184)$ & $666(164)$ & $626(180)$ \\
\hline Right key & $665(164)$ & $661(129)$ & $671(202)$ & $640(142)$ & $670(213)$ & $573(196)$ \\
\hline Experiment 4 & $\mathrm{~L}-\mathrm{L}$ & $\mathrm{C}-\mathrm{C}$ & $\mathrm{R}-\mathrm{R}$ & $\mathrm{L}-\mathrm{L}$ & $\mathrm{C}-\mathrm{C}$ & $\mathrm{R}-\mathrm{R}$ \\
\hline Left key & $510(168)$ & $555(152)$ & $545(160)$ & $599(142)$ & $617(185)$ & 609 (223) \\
\hline Right key & $581(159)$ & $607(165)$ & $592(180)$ & $560(162)$ & $570(147)$ & 499 (102) \\
\hline
\end{tabular}

with the intermediate-coding account (Kong \& You, 2012). In our temporal estimation task, the physical space and the manual response codes were involved in the task and influenced the space-time compatibility effect. In other words, the physical, spatial, and manual response codes were independent in associating short/long durations with left/right keys, such as associating temporal durations with $\mathrm{C}-\mathrm{L} / \mathrm{C}-\mathrm{R}$ conditions.

However, the results of NEs showed a STEARC effect but not a durations by stimuli locations congruency effect, indicating that the motor component was more relevant to the task than the spatial processing of target position. It is worth noting that a Simon-like effect (Simon, 1990) was also found, indicating that the spatial position of the target (which was irrelevant to the task) not only was processed, but also affected the performance accuracy.
As was noted by Di Bono et al. (2012), the results could be confounded by the fact that the spatial and temporal dimensions were simultaneously conveyed within the same target stimuli. The independent congruency effect of perceptual and motor components in spatial-temporal association could be explained considering that the external physical space codes were linked to the spatial positions of the target, which provided the relevant temporal information to perform the task in our experimental procedure. However, the duration of the reference stimulus also had to be estimated by the participants in order to perform the task. Thus, the external physical space codes could be linked to the spatial position of the reference stimulus. In order to test this possibility, in Experiment 2 the reference stimulus was spatially varied while the target stimuli remained fixed in the central position. Thus, the spatial dimension was conveyed by the

Table 2 Mean numbers of errors (and standard deviations) for left and right keys when judging short and long durations, for each spatial condition in all experiments

\begin{tabular}{|c|c|c|c|c|c|c|}
\hline \multirow[b]{2}{*}{ Experiment 1} & \multicolumn{3}{|c|}{ Short Durations } & \multicolumn{3}{|c|}{ Long Durations } \\
\hline & $\mathrm{C}-\mathrm{L}$ & $\mathrm{C}-\mathrm{C}$ & $\mathrm{C}-\mathrm{R}$ & $\mathrm{C}-\mathrm{L}$ & $\mathrm{C}-\mathrm{C}$ & $\mathrm{C}-\mathrm{R}$ \\
\hline Left key & $0.35(0.63)$ & $0.68(0.73)$ & $0.68(0.99)$ & $2.25(1.49)$ & $2.90(1.95)$ & 2.35 (1.47) \\
\hline Right key & $1.18(0.96)$ & $0.70(0.66)$ & $0.68(0.61)$ & $2.10(1.29)$ & $2.70(1.46)$ & $1.67(1.25)$ \\
\hline Experiment 2 & $\mathrm{~L}-\mathrm{C}$ & $\mathrm{C}-\mathrm{C}$ & $\mathrm{R}-\mathrm{C}$ & $\mathrm{L}-\mathrm{C}$ & $\mathrm{C}-\mathrm{C}$ & $\mathrm{R}-\mathrm{C}$ \\
\hline Left key & $0.50(0.46)$ & $0.43(0.63)$ & $0.65(0.59)$ & $1.20(0.98)$ & $1.30(1.02)$ & $1.22(0.80)$ \\
\hline Right key & $0.50(0.49)$ & $0.38(0.46)$ & $0.53(0.47)$ & $1.35(0.99)$ & $1.23(0.90)$ & $1.38(0.81)$ \\
\hline Experiment 3 & $\mathrm{R}-\mathrm{L}$ & $\mathrm{C}-\mathrm{C}$ & $\mathrm{L}-\mathrm{R}$ & $\mathrm{R}-\mathrm{L}$ & $\mathrm{C}-\mathrm{C}$ & $\mathrm{L}-\mathrm{R}$ \\
\hline Left key & $0.43(0.61)$ & $0.23(0.38)$ & $0.43(0.63)$ & $0.53(0.88)$ & $0.63(0.78)$ & $0.43(0.65)$ \\
\hline Right key & $0.48(0.60)$ & $0.53(0.62)$ & $0.60(0.68)$ & $0.63(0.78)$ & $0.48(0.66)$ & $0.48(0.73)$ \\
\hline Experiment 4 & $\mathrm{~L}-\mathrm{L}$ & $\mathrm{C}-\mathrm{C}$ & $\mathrm{R}-\mathrm{R}$ & $\mathrm{L}-\mathrm{L}$ & $\mathrm{C}-\mathrm{C}$ & $\mathrm{R}-\mathrm{R}$ \\
\hline Left key & $0.72(0.64)$ & $0.45(0.43)$ & $0.70(0.59)$ & $0.92(0.92)$ & $0.75(0.73)$ & 0.85 (1.09) \\
\hline Right key & $0.90(0.93)$ & $1.00(0.99)$ & $0.63(0.63)$ & $0.40(0.64)$ & $0.28(0.44)$ & $0.45(0.36)$ \\
\hline
\end{tabular}


reference, whereas the temporal dimension was mainly conveyed by the (central) target.

\section{Experiment 2}

In Experiment 2, the position of the reference stimulus was varied on the screen, while the target stimuli remained fixed in the central position. The participants had to perform the same temporal estimation task as in Experiment 1. The interaction between response keys and duration judgment (short-long) should indicate a STEARC effect, supporting a motor contribution for the spatial-temporal association. The interaction between duration judgment and spatial condition should indicate a Duration $\times$ Stimulus Location congruency effect, supporting a perceptual contribution to the spatial-temporal association. Finally, the triple interaction should indicate a modulation of the congruency effect, as explained in Experiment 1.

\section{Method}

Participants A group of 20 university students (14 females, six males) volunteered to participate in the study. Their mean age was 26.95 years $(S D=6.71)$. According to the EHI scores (Oldfield, 1971), 16 were right-handed and four were left-handed. All of the participants had normal or correctedto-normal vision, and none had participated in Experiment 1. The study was conducted following university ethical guidelines, and all participants provided written informed consent.

Materials The materials were the same as in Experiment 1, with the following exception. The reference stimuli appeared in the left, central, or right position on the screen, whereas the target stimuli appeared in the central position for each trial. Thus, the reference-target pair determined three spatial positions: (1) left-center ( $\mathrm{L}-\mathrm{C}$ ) position, (2) center-center (C-C) position, or (3) right-center $(\mathrm{R}-\mathrm{C})$ position. The $x$ and $y$-coordinates for the left, center, and right positions were the same as in Experiment 1.

Procedure The task and procedure were the same as in Experiment 1, with a fixed duration $(400 \mathrm{~ms})$ of the reference stimulus and a target stimulus that randomly lasted for 200 , 300,500 , or $600 \mathrm{~ms}$.

Data analysis The mean RTs of correct responses was calculated. Moreover, RTs more than 3 SDs above and below the mean were excluded from the analysis because they were considered outliers (about $1.5 \%$ of the original sample). For RTs as well as NEs, we followed the same data analysis described in Experiment 1. Thus, three-way repeated measures ANOVAs were carried out separately on RTs and NEs, with Key (two levels: left vs. right), Duration (two levels: short vs. long), and Condition (two levels: L-C vs. R-C) as within-subjects factors. When a reliable and significant effect was found, the Scheffé post-hoc test was run. Values with $p<.05$ were considered significant.

\section{Results and discussion}

Table 1 reports the mean RTs for the spatial response keys and temporal durations in all spatial conditions.

The results of the ANOVA ${ }^{2}$ on RTs mirrored those of Experiment 1. Indeed, we found a significant Key $\times$ Duration interaction $\left[F(1,19)=11.44, p<.005, \eta_{\mathrm{p}}{ }^{2}=.38\right]$. A Scheffé post-hoc test showed that the long durations were responded to faster with the right key $(571 \mathrm{~ms}, S D=57 \mathrm{~ms})$ than with the left key $(607 \mathrm{~ms}, S D=62 \mathrm{~ms})$, with $p<.05$. No other comparisons were significant. Additionally, the Duration $\times$ Condition interaction reached significance $[F(1,19)=4.58$, $\left.p<.05, \eta_{\mathrm{p}}{ }^{2}=.19\right]$ : When the reference stimulus appeared on the left side of the screen, short durations $(577 \mathrm{~ms}, S D=$ $57 \mathrm{~ms}$ ) of the target were judged faster than long durations (597 ms, $S D=65 \mathrm{~ms}$ ), although this comparison was not significant. When the reference cue appeared on the right of the screen, long durations were judged faster $(581 \mathrm{~ms}, S D=$ $54 \mathrm{~ms}$ ) than short durations $(592 \mathrm{~ms}, S D=46 \mathrm{~ms})$. No main effects $(F \mathrm{~s}<2.45, p \mathrm{~s}>.13)$ or other interactions (Key $\times$ Condition, $p=.08)$ were significant. Of greater interest, we did not find a significant triple interaction $(F \approx 0)$.

In Table 2, the mean NEs for the spatial response keys and temporal durations in all spatial conditions are shown.

The ANOVA on NEs revealed a significant duration effect only $\left[F(1,19)=13.63, p<.005, \eta_{\mathrm{p}}{ }^{2}=.42\right]$, with lower NEs for short $(0.54, S D=0.50)$ than for long $(1.29, S D=0.90)$ durations. No other main effects or interactions were statistically significant $\left(F_{\mathrm{S}}<1.30, p \mathrm{~s}>.25\right)$.

The main results mirrored those of Experiment 1 . On the one hand, we observed a STEARC effect, suggesting that the spatial representation of time was influenced by the spatial position of the response buttons. On the other hand, we found a Duration $\times$ Stimulus Location congruency effect, suggesting that the spatial representation of time was also

\footnotetext{
${ }^{2}$ The overall ANOVA on RTs, with Key (two levels: left vs. right), Duration (two levels: short vs. long), and Condition (three levels: C-L vs. $\mathrm{C}-\mathrm{C}$ vs. $\mathrm{C}-\mathrm{R}$ ) as within-subjects factors, revealed a significant condition effect $\left[F(1.917,36.420)=10.52, p<.0001, \eta_{\mathrm{p}}{ }^{2}=.36\right.$, with GreenhouseGeisser correction]. The $\mathrm{C}-\mathrm{C}$ condition $(611 \mathrm{~ms}, S D=45 \mathrm{~ms})$ elicited higher RTs than did the $\mathrm{L}-\mathrm{C}$ and $\mathrm{R}-\mathrm{C}$ conditions. The Condition factor did not interact with any other factors $\left(F_{\mathrm{S}}<2.56, p \mathrm{~s}>.10\right)$, whereas we did find a significant Key $\times$ Duration interaction similar to the one reported in the text.

The same ANOVA on NEs did not show any significant condition effect, and this factor did not interact with other factors $\left(F_{\mathrm{S}}<1.40, p \mathrm{~s}>.20\right)$. The duration effect reported in the text remained reliable.
} 
influenced by the spatial position of the reference stimulus. This finding is relevant, considering that the spatial (i.e., where the reference appeared) and temporal dimensions were not simultaneously conveyed within the same stimuli, in line with Di Bono et al. (2012). In addition, no triple interaction was found, reflecting that the motor and perceptual components were independent in their time-space associations (Ouellet et al., 2010; Santiago et al., 2007).

In both of these experiments, the source of the perceptual component was related to only one type of stimulus: the target position in Experiment 1, and the reference position in Experiment 2. Vicario et al. (2008) asked participants to perform a temporal estimation task with reference and target stimuli (i.e., black dots) and spatially coded responses. In particular, three spatial conditions were created: the reference stimulus appeared on the left side of the space while the target stimulus appeared on the right; the reference stimulus appeared on the right while the target stimulus appeared on the left; and both the reference and target stimuli appeared at the center of the screen. A strong influence of spatial lateralization on time judgments was found: Left space biased the estimations toward short durations, whereas right space biased the estimations toward long durations. Thus, the results of Vicario et al. (2008) study could be discussed in terms of the temporal information "moving" in a perceptual aspect from left to right and from right to left. Nevertheless, Vicario et al. (2008) did not analyze the influence of response codes on time judgments. Consequently, it is not clear whether the associations between left space and short durations, as well as between right space and long durations, were influenced by the experimental presentation of the stimuli or by the spatial position of the response buttons.

In Experiment 3, both stimuli were presented in opposite positions (e.g., the reference appeared on the left, and its associated target appeared on the right of the screen). In addition, two spatial response buttons were pressed. In this way, we could test whether the time-space association was related to perceptual or motor components.

\section{Experiment 3}

In Experiment 3, the reference and the target were presented in opposite spatial positions. For example, the reference appeared on the left, and then the subsequent target appeared on the right. The reversed manipulation was carried out for a right reference and then a left target. Alternatively, the spatial information could remain fixed, with both temporal stimuli being presented in the central position. According to the spatial positions of the reference and the target, movement from left to right space and movement from right to left space was created. In this case, spatial information for temporal stimuli was provided by the reference-target pair, whereas the spatial information for the manual responses was provided by the left/right keys. As we previously described, the STEARC effect should indicate the influence of the motor component, whereas the compatibility between the spatial positions of the stimuli with the temporal duration should indicate the influence of the perceptual component. An interaction between spatial response keys, spatial positions of the stimuli, and the temporal duration to be judged should indicate a modulation of the congruency effect.

\section{Method}

Participants A group of 20 university students (13 females, seven males) volunteered to participate in the study. Their mean age was 25.25 years $(S D=7.35)$. According to the EHI score (Oldfield, 1971), 18 were right-handed and two were left-handed. All of the participants had normal or correctedto-normal vision, the none had participated in the previous experiments in this study. The study was conducted following university ethical guidelines, and all participants provided written informed consent.

Materials The materials were the same as in Experiments 1 and 2, with the following exceptions. The reference-target pair determined three spatial positions: (1) left-right (L-R) position, (2) center-center $(\mathrm{C}-\mathrm{C})$ position, or (3) right-left (R-L) position. The $x$ - and $y$-coordinates for left, center, and right positions were the same as were used in Experiments 1 and 2.

Procedure The procedure was the same as in Experiments 1 and 2.

Data analysis Mean RTs of the correct responses were calculated. Moreover, RTs more than $3 S D$ s above or below the mean were excluded from the analysis because they were considered outliers (about $2.3 \%$ of the original sample). For RTs as well as for NEs, we followed the same data analysis procedure as was described in Experiment 1. A three-way repeated measures ANOVA was carried out, with Key (two levels: left vs. right), Duration (two levels: short vs. long), and Condition (two levels: L-R vs. R-L) as within-subjects factors. When a reliable and significant effect was found, the Scheffé post-hoc test was run. Values with $p<.05$ were considered to be significant.

\section{Results and discussion}

Table 1 reports the mean RTs for the spatial response keys and temporal durations in all spatial positions of each reference-target pair. 
The ANOVA ${ }^{3}$ on RTs mirrored those for Experiments 1 and 2 , with a significant interaction between the Key and Duration factors $\left[F(1,19)=15.11, p<.001, \eta_{\mathrm{p}}{ }^{2}=.44\right]$. A post-hoc test showed that for the short duration the left key $(597 \mathrm{~ms}, S D=$ $164 \mathrm{~ms}$ ) was faster than the right key (668 ms, $S D=183 \mathrm{~ms})$, with $p<.05$. In addition, the right key was pressed more quickly for long durations (606 ms, $S D=169 \mathrm{~ms}$ ) than for short durations, with $p<.05$. The Duration $\times$ Condition interaction was also significant $\left[F(1,19)=4.43, p<.05, \eta_{\mathrm{p}}{ }^{2}=.19\right]$. In the Scheffé test, no significant comparisons were found, even if the R-L condition ( $622 \mathrm{~ms}, S D=165 \mathrm{~ms}$ ) did induce faster responses than the $\mathrm{L}-\mathrm{R}$ condition $(642 \mathrm{~ms}, S D=182 \mathrm{~ms}$ ) for short durations, and the reverse was observed for long durations. No main effects or other interactions were significant $\left(F_{\mathrm{S}}<2.20, p \mathrm{~s}>.12\right)$. As in previous experiments, no congruency effect was found.

In Table 2, the mean NEs for the spatial response keys and temporal durations at all spatial positions of the referencetarget pair are shown.

The aforementioned ANOVA on NEs did not yield any significant main effects or interactions $(F \mathrm{~s}<1.60, p \mathrm{~s}>.20)$.

The results of this experiment mirrored those of Experiments 1 and 2: The spatial-temporal association derived from the influence of both perceptual (Di Bono et al., 2012; Vicario et al., 2008) and motor (Fabbri et al., 2012; Ishihara et al., 2008; Vallesi et al., 2008) components. The present experiment extended the study of Vicario et al. (2008), suggesting that not only are the $\mathrm{L}-\mathrm{R}$ and $\mathrm{R}-\mathrm{L}$ conditions affected the time estimation, but also by the spatial positions of the response keys. However, we did not show a significant triple interaction, indicating that the perceptual and motor components were independent of time-space compatibility.

In Experiment 4, the reference-target pair created three spatial positions at which the reference and target stimuli would both appear (e.g., the reference appeared on the left, and then the subsequent target appeared on the left). Thus, both temporal stimuli coded the same spatial position, and it was likely that the physical spatial codes would be maximized.

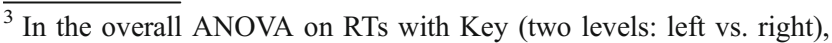
Duration (two levels: short vs. long), and Condition (three levels: $\mathrm{C}-\mathrm{L}$ vs. $\mathrm{C}-\mathrm{C}$ vs. $\mathrm{C}-\mathrm{R}$ ) as within-subjects factors, we found a significant condition effect $\left[F(1.882,35.765)=3.60, p<.05, \eta_{\mathrm{p}}{ }^{2}=.16\right.$, with Greenhouse-Geisser correction]. The $\mathrm{C}-\mathrm{C}$ condition $(661 \mathrm{~ms}, S D=$ $170 \mathrm{~ms}$ ) had higher RTs than did the other conditions ( $p \mathrm{~s}<.05$ for both comparisons). Similarly to the results reported in the text, the STEARC effect was significant. In contrast, the Duration $\times$ Condition interaction was not significant $(F<2.50, p>.10)$, whereas the triple interaction reached a significant level $\left[F(1.691,32.124)=4.34, p<.05, \eta_{\mathrm{p}}{ }^{2}=.19\right.$, with Greenhouse-Geisser correction]. These different results could be explained by considering that in the $\mathrm{C}-\mathrm{C}$ conditions the participants were slower (Table 1 ) than in the $\mathrm{L}-\mathrm{R}$ and $\mathrm{R}-\mathrm{L}$ conditions.

The same ANOVA on NEs confirmed the lack of significant effects or interactions, as is reported in the text.
}

\section{Experiment 4}

In Experiment 4, both the reference and the target spatially varied on the screen, but in the same position as one another within the same trial. For example, the reference appeared on the left side of the screen, and then the subsequent target also appeared on the left side of the screen. The same manipulation was carried out for the central and right spaces. Thus, the spatial information was similar in both temporal stimuli. The STEARC effect should indicate the influence of the motor component, whereas the compatibility of the spatial positions of the stimuli with the temporal duration should indicate the influence of the perceptual component. An interaction between the spatial response keys, spatial positions of the stimuli, and the temporal duration to be judged should indicate a modulation of the congruency effect when both components were congruent.

\section{Method}

Participants A group of 20 university students (16 females, four males) volunteered to participate in this study. Their mean age was 24.60 years $(S D=3.94)$. According to the EHI scores (Oldfield, 1971), 15 were right-handed and five were left-handed. All participants had normal or corrected-tonormal vision, and none had participated in the previous experiments of this study. The study was conducted following university ethical guidelines, and all participants provided written informed consent.

Materials The materials were the same as in Experiment 3, with the following exception. The reference stimuli were presented at the left, central, or right positions on the screen, as were the corresponding target stimuli. Thus, the reference-target pair determined three spatial positions: (1) leftleft ( $\mathrm{L}-\mathrm{L}),(2)$ center-center $(\mathrm{C}-\mathrm{C})$, or (3) right-right (R-R) positions. The $x$ - and $y$-coordinates for the left, center, and right positions were the same as those used in Experiments 1 and 2.

Procedure The procedure was the same as in previous experiments.

Data analysis The mean RTs of correct responses were calculated. Moreover, RTs more than $3 S D$ above or below the mean were excluded from the analysis because they were considered outliers (about $1.8 \%$ of the original sample). For RTs as well as NEs, we followed the same data analysis procedure described in Experiment 1. A three-way repeated measures ANOVA was carried out, with Key (two levels: left vs. right), Duration (two levels: short vs. long), and Condition (two levels: L-L vs. R-R) as within-subjects factors. When a reliable and significant effect was found, a Scheffé 
post-hoc test was run. Values with $p<.05$ were considered to be significant.

\section{Results and discussion}

Table 1 reports the mean RTs for the spatial response keys and temporal durations in all spatial positions of the reference-target pairs.

The ANOVA ${ }^{4}$ on RTs showed that the Key factor significantly interacted with the Duration $[F(1,19)=8.77, p<.05$, $\left.\eta_{\mathrm{p}}{ }^{2}=.32\right]$ and Condition $\left[F(1,19)=5.22, p<.05, \eta_{\mathrm{p}}{ }^{2}=.22\right]$ factors. Regarding the former interaction, a STEARC effect was found, suggesting that the left key ( $527 \mathrm{~ms}, S D=164 \mathrm{~ms})$ was pressed more quickly than the right key $(587 \mathrm{~ms}, S D=$ $170 \mathrm{~ms}$ ) when detecting short durations $(p<.05)$, whereas the right key (529 ms, $S D=132 \mathrm{~ms}$ ) was pressed more quickly than the left key (604 ms, $S D=183 \mathrm{~ms})$ when detecting long durations $(p<.05)$. For the latter interaction, the post-hoc test did not show any significant comparisons, even if the right key did show lower RTs for the R-R condition $(545 \mathrm{~ms}, S D=$ $141 \mathrm{~ms}$ ) than for the $\mathrm{L}-\mathrm{L}$ condition $(571 \mathrm{~ms}, S D=161 \mathrm{~ms})$. The reverse situation was found for the left key in the $\mathrm{L}-\mathrm{L}$ condition (554 ms, $S D=155 \mathrm{~ms})$ with respect to the R-R condition ( $577 \mathrm{~ms}, S D=191 \mathrm{~ms})$. The ANOVA also showed a significant Duration $\times$ Condition interaction $[F(1,19)=5.75$, $\left.p<.05, \eta_{\mathrm{p}}{ }^{2}=.23\right]$, reflecting a Duration $\times$ Stimulus Location congruency effect. Although none of the post-hoc comparisons were significant, the $\mathrm{L}-\mathrm{L}$ condition $(546 \mathrm{~ms}, S D=$ $163 \mathrm{~ms}$ ) was faster than the R-R condition $(568 \mathrm{~ms}, S D=$ $170 \mathrm{~ms}$ ) for short durations, whereas the R-R condition (554 ms, $S D=162 \mathrm{~ms}$ ) was associated with faster responses than the $\mathrm{L}-\mathrm{L}$ condition $(580 \mathrm{~ms}, S D=152 \mathrm{~ms}$ ) for long durations. No main effects were significant, nor the triple interaction between the Key, Duration, and Condition factors $\left(F_{\mathrm{s}}<1.00, p \mathrm{~s}>.40\right)$.

In Table 2, the mean NEs for spatial response keys and temporal durations in all spatial positions of the referencetarget pairs are shown.

The aforementioned ANOVA on NEs did not yield any significant main effects (key, $p=.07$; duration, $p=.64$; and condition, $p=.27)$ or interactions $\left(F_{\mathrm{s}}<2.72, p \mathrm{~s}>.11\right)$.

The results of Experiment 4 confirmed that both STEARC (Fabbri et al., 2012; Ishihara et al., 2008; Vallesi et al., 2008) and Duration $\times$ Stimulus Location congruency (Di Bono

\footnotetext{
${ }^{4}$ The overall ANOVA on RTs showed a significant condition effect $\left[F(1.784,33.892)=5.38, p<.05, \eta_{\mathrm{p}}{ }^{2}=.22\right.$, with Greenhouse-Geisser correction], reflecting slower RTs in the $\mathrm{C}-\mathrm{C}$ condition $(587 \mathrm{~ms}, 163$ $\mathrm{ms}$ ) than in the $\mathrm{L}-\mathrm{L}$ and $\mathrm{R}-\mathrm{R}$ conditions ( $p \mathrm{~s}<.05$ for both comparisons). The significant Key $\times$ Duration interaction remained reliable, but no other interactions were found $(F<2.10, p>.10)$, in contrast to the results in the text. As before, the condition effect could influence the results (Table 1).

The same ANOVA on NEs showed a significant Key $\times$ Duration interaction, but no condition effect or interactions $(F \mathrm{~s}<2.50, p \mathrm{~s}>.11)$.
}

et al., 2012; Ouellet et al., 2010; Santiago et al., 2007) effects were found, and they did not significantly interact. Even though the reference-target pair conveyed the same spatial information (in part explaining the detected Simon-like effect), no facilitation effect was found when both the motor and perceptual components were congruent (i.e., left key, left position at short durations and right key, right position at long durations). This pattern of results was similar across experiments, apart from the spatial manipulation of the temporal stimuli. Thus, the task characteristics could induce the spatial-temporal associations, considering either the spatial lateralization of response keys or the spatial positions of the temporal stimuli.

\section{General discussion}

The goal of the present study was to investigate the influence of perceptual and motor components in space-time interactions using a visual temporal estimation task with two lateralized response buttons. In other words, we studied whether, in the spatial-temporal association, the motor and perceptual components were independent from each other or induced a modulation of the congruency effect when both components were congruent.

The results of all of our experiments were clear cut. First, a clear STEARC effect was found (Fabbri et al., 2012; Ishihara et al., 2008; Vallesi et al., 2008). The involvement of two lateralized response keys (motor component) affected the performance. Specifically, participants were faster if they judged short durations with the left key (hand) and long durations with the right key (hand) than vice versa. This pattern of results suggests that elapsing time is internally mapped onto a left-to-right mental time line (Arzy et al., 2009; Bonato et al., 2012), extending the findings when temporal words (past or future) have been used (Rolke et al., 2013; Torralbo et al., 2006; Weger \& Pratt, 2008; with auditory stimuli, see Ouellet, Román, \& Santiago, 2012). The left-short and right-long associations observed in the present study are in line with Proctor and Cho's (2006) polarity account, according to which salient stimulus features are mapped onto salient response codes. The STEARC effect could be considered an instance of a polarity correspondence principle, assuming that time (short $[-]$ and long $[+]$ ) and response (left $[-]$ and right $[+]$ ) are coded on a bipolar dimension and that corresponding polarities induce faster response selection (Proctor \& Cho, 2006). Thus, the STEARC effect may be due to the polarity correspondence between durations and responses. With right and long being the unmarked poles and left and short being the marked poles, the left-short and right-long associations are shown. In recent work, Vallesi, McIntosh, and Stuss (2011) showed that the spatial representation of time produced a response code that preactivated the corresponding motor cortex and sped up the response when short and long durations were 
mapped onto left and right keys, respectively. The same result was not found for incompatible mappings, which produced a cost in terms of speed (Vallesi et al., 2011). Consequently, the STEARC effect found here is evidence for the role of the motor components in space-time interaction.

Secondly, a Duration $\times$ Stimulus Location congruency effect was observed (Di Bono et al., 2012; Ouellet et al., 2010; Santiago et al., 2007; Vicario et al., 2008; with auditory stimuli see Lakens, Semin, \& Garrido, 2011). The manipulation across experiments of reference-target pairs affected the performance. Even if the left or right position of the stimuli was taskirrelevant, participants were faster to judge short durations when the reference (Exps. 2 and 4) and/or the target (Exps. 1 and 3) appeared in the left space, and to judge long durations when the reference/target appeared in right space than in the reversed situations. This congruency effect between the durations and spatial positions of stimuli is consistent with a leftshort and right-long mental representation of time (Bonato et al., 2012). Here, we found a congruency effect using rectangles as temporal stimuli. The same effect has also been found when temporal words, such as past or future, were used. This finding can be discussed in terms of the orientation of visuospatial attention to left or right space according to the temporal stimuli (past or future words, respectively), as was found by Ouellet et al. (2010). In line with this assumption, Frassinetti, Magnani, and Oliveri (2009) found temporal underestimation and overestimation after prismatic deviation. In other words, the orienting of visuospatial attention might enhance left-right physical space, which, in turn, might enhance the corresponding side of temporal space. Similarly, Basso, Nichelli, Frassinetti, and di Pellegrino (1996) found that a neglect patient overestimated the durations of stimuli presented in the leftward part of right space and underestimated the durations of stimuli presented in the nonneglected field.

Of greater interest, we did not find a significant interaction between the Key, Duration, and Condition factors in any experiment, and thus found no modulation of the congruency effects when both the motor and perceptual components were congruent. This finding suggests that the motor and perceptual components are independent from each other for space-time interaction. In addition, we obtained faster RTs when there was a congruency between a left key, left space (i.e., $\mathrm{C}-\mathrm{L}, \mathrm{L}-\mathrm{C}, \mathrm{R}-\mathrm{L}$, and $\mathrm{L}-\mathrm{L}$ for each experiment, respectively), and short durations, as well as between a right key, right space (i.e., $\mathrm{C}-\mathrm{R}, \mathrm{R}-\mathrm{C}, \mathrm{L}-\mathrm{R}$, and $\mathrm{R}-\mathrm{R}$ ), and long durations (Table 1). These results add evidence to the idea that we obtained two independent (or additive) congruency effects. As was noted by Santiago et al. (2007) and Ouellet et al. (2010), the spatial-temporal association was based not only on the priming of manual response codes, but also on physical space codes. This result could be accounted for (and extended to) the intermediate-coding account proposed by Kong and You (2012). The authors proposed that temporal meanings activate an abstract spatial code, such as "left" or "right." The association between temporal meanings and spatial codes was preexisting in long-term memory. The crucial point of this account is that these spatial codes could be associated with manual response and physical space codes, according to the task characteristics. Thus, "task characteristics have an important impact on the use of mental strategy" (Kong \& You, 2012, p. 86). In our task, both physical space and manual response codes were involved in the task at the same time, and thus, abstract spatial codes could activate both physical spatial codes and manual response codes, in a way similar to in the study by Ouellet et al. (2010).

This last assumption could be supported by the fact that Fabbri et al. (2013) only obtained a STEARC effect in a temporal reproduction task with the same spatial manipulation of temporal stimuli and response keys. The lack of any spatialtemporal congruency effect could be due to the difference between temporal estimation (used here) and reproduction tasks (Fabbri et al., 2013). These two tasks are directly comparable, as they share common encoding and storing of temporal information, but they differ in how participants use the time information to make responses (Bueti \& Walsh, 2010; Bueti, Walsh, Frith, \& Rees, 2008). In the time estimation task, the motor response is required in order to make an estimation (i.e., shorter-longer), whereas in the time reproduction task, the motor response is required in order to reproduce an estimated duration, and thus action duration is coded as part of a motor program and can be processed prior to motor execution. Thus, in the temporal reproduction task, in which temporal judgments are based primarily on motor rather than perceptual representations (Bueti \& Walsh, 2010; Bueti et al., 2008; Fabbri et al., 2012), when manual responses and physical spatial codes are involved in the task at the same time, the motor components seem to mask (or reduce) the contribution of perceptual components. This is not the case in the temporal estimation task, in which the perceptual component contributes to the space-time interaction.

To sum up, in a time estimation task with lateralized responses and manipulation of the spatial positions of temporal stimuli, the motor and perceptual components were independent from each other for space-time association. The leftto-right mapping of time representations could depend on the spatial information provided by spatial response keys and by the spatial positions of the stimuli. Thus, the motor and perceptual compatibility effects could arise and be better explained by the intermediate-coding account.

\section{References}

Arzy, S., Adi-Japha, E., \& Blanke, O. (2009). The mental time line. An analogue of the mental number line in the mapping of life events. Consciousness and Cognition, 18, 781-785. doi:10.1016/j.concog. 2009.05.007 
Basso, G., Nichelli, P., Frassinetti, F., \& di Pellegrino, G. (1996). Time perception in a neglected space. NeuroReport, 7, 2111-2114.

Bonato, M., Zorzi, M., \& Umiltà, C. (2012). When time is space: Evidence for a mental time line. Neuroscience and Biobehavioral Reviews, 36, 2257-2273. doi:10.1016/j.neubiorev.2012.08.007

Bueti, D., \& Walsh, V. (2010). Memory for time distinguishes between perception and action. Perception, 39, 81-90. doi:10.1068/p6405

Bueti, D., Walsh, V., Frith, C., \& Rees, G. (2008). Different brain circuits underlie motor and perceptual representations of temporal intervals. Journal of Cognitive Neuroscience, 20, 204-214. doi:10. 1162/jocn.2008.20.2.204

Cappelletti, M., Freeman, E. D., \& Cipolotti, L. (2009). Dissociation and interactions between time, numerosity and space processing. Neuropsychologia, 47, 2732-2748. doi:10.1016/j.neuropsychologia. 2009.0.024

Di Bono, M. G., Casarotti, M., Priftis, K., Gava, L., Umiltà, C., \& Zorzi, M. (2012). Priming the mental time line. Journal of Experimental Psychology. Human Perception and Performance. doi:10.1037/ a0028346

Fabbri, M., Cancellieri, J., \& Natale, V. (2012). The A Theory of Magnitude (ATOM) model in temporal perception and reproduction tasks. Acta Psychologica, 139, 111-123. doi:10.1016/j.actpsy. 2011.09.006

Fabbri, M., Cellini, N., Martoni, M., Tonetti, L., \& Natale, V. (2013). The mechanisms of space-time association: comparing motor and perceptual contributions in time reproduction. Cognitive Science. doi:10.1111/cogs. 12038

Frassinetti, F., Magnani, B., \& Oliveri, M. (2009). Prismatic lenses shift time perception. Psychological Science, 20, 949-954. doi:10. 1111/j.1467-9280.2009.02390.x

Ishihara, M., Keller, P. E., Rossetti, Y., \& Prinz, W. (2008). Horizontal spatial representation of time: Evidence for the STEARC effect. Cortex, 44, 454-461. doi:10.1016/j.cortex.2007.08.010

Kong, F., \& You, X. (2012). Space-time compatibility effects in the auditory modality. Experimental Psychology, 59, 82-87.

Lakens, D., Semin, G. R., \& Garrido, M. V. (2011). The sound of time: Cross-modal convergence in the spatial structuring of time. Consciousness and Cognition, 20, 437-443. doi:10.1016/j.concog. 2010.09.020

Lakoff, G., \& Johnson, M. (1980). Metaphors we live by. Chicago, IL: University of Chicago Press.

Lakoff, G., \& Johnson, M. (1999). Philosophy in the flesh: The embodied mind and its challenge to Western thought. New York, NY: Basic Books.

Lewis, P. A., \& Miall, R. C. (2006). Remembering the time: A continuous clock. Trends in Cognitive Sciences, 10, 401-406. doi:10. 1016/j.tics.2006.07.006

Oldfield, R. C. (1971). The assessment and analysis of handedness: The Edinburgh inventory. Neuropsychologia, 9, 97-113.

Ouellet, M., Román, A., \& Santiago, J. (2012). A multisensory interaction effect in the conceptual realm of time. Experimental Psychology, 59, 236-242. doi:10.1027/1618-3169/a000148

Ouellet, M., Santiago, J., Funes, M. J., \& Lupiáñez, J. (2010). Thinking about the future moves attention to the right. Journal of Experimental
Psychology. Human Perception and Performance, 36, 17-24. doi:10. 1037/a0017176

Proctor, R. W., \& Cho, Y. S. (2006). Polarity correspondence: A general principle for performance of speeded binary classification tasks. Psychological Bulletin, 132, 416-442. doi:10.1037/0033-2909. 132.3.416

Radden, G. (2004). The metaphor TIME AS SPACE across languages. In N. Baumgarten, C. Bottger, M. Moltz, \& J. Probst (Eds.), Uebersetzen, interkulturelle Kommunikation, Spracherwerb und Sprachvermittlung - das Leben mit mehreren sprachen: Festschrift fuer Juliane House zum 60. Geburtstang (pp. 226-239). Bochum: Aks-Verlag.

Rolke, B., Fernández, S. R., Schmid, M., Walker, M., Lachmair, M., López, J. J. R., \& Vázquez, C. (2013). Priming the mental timeline: effects of modality and processing mode. Cognitive Processing. doi:10.1007/s10339-013-0537-5

Santiago, J., Lupiáñez, J., Perez, E., \& Funes, M. J. (2007). Time (also) flies from left to right. Psychonomic Bulletin \& Review, 14, 512516.

Santiago, J., Román, A., Ouellet, M., Rodríguez, N., \& Pérez-Azor, P. (2010). In hindsight, life flows from left to right. Psychological Research, 74, 59-70. doi:10.1007/s00426-008-0220-0

Schneider, W., Eschman, A., \& Zuccolotto, A. (2002). E-Prime user's guide. Pittsburgh, PA: Psychology Software Tools Inc.

Simon, J. R. (1990). The effects of an irrelevant directional cue on human information processing. In R. Proctor \& T. Reeve (Eds.), Stimulus-response compatibility: An integrated perspective (pp. 31-86). Amsterdam, The Netherlands: Elsevier Science.

Torralbo, A., Santiago, J., \& Lupiáñez, J. (2006). Flexible conceptual projection of time onto spatial frames of reference. Cognitive Science, 30, 749-757.

Tversky, B., Kugelmass, S., \& Winter, A. (1991). Cross-cultural and developmental trends in graphic productions. Cognitive Psychology, 23, 515-557.

Vallesi, A., Binns, M. A., \& Shallice, T. (2008). An effect of spatialtemporal association of response codes: Understanding the cognitive representations of time. Cognition, 107, 501-527. doi:10. 1016/j.cognition.2007.10.011

Vallesi, A., McIntosh, A. R., \& Stuss, D. T. (2011). How time modulates spatial responses. Cortex, 47, 148-156. doi:10.1016/j.cortex. 2009.09.005

Vicario, C. M., Pecoraro, P., Turriziani, P., Koch, G., Caltagirone, C., \& Oliveri, M. (2008). Relativistic compression and expansion of experimental time in the left and right space. PLoS One, 3, e1716. doi:10.1371/journal.pone.0001716

Vicario, C. M., Rappo, G., Pepi, A. M., \& Oliveri, M. (2009). Timing flickers across sensory modalities. Perception, 38, 1144-1151. doi: $10.1068 / \mathrm{p} 6362$

Vidal, F., Bonnet, M., \& Macar, F. (1991). Programming response duration in a precueing reaction time paradigm. Journal of Motor Behavior, 23, 226-234. doi:10.1080/00222895.1991.9942033

Weger, U., \& Pratt, J. (2008). Time flies like an arrow: Shifting spatial attention in response to adverbs of time. Psychonomic Bulletin \& Review, 15, 426-430. doi:10.3758/PBR.15.2.426 